RESEARCH ARTICLE

\title{
Angiographic Pattern of Coronary Artery Disease in Diabetic Patients Having Abnormal Ankle Brachial Index
}

\author{
Syed Haseeb Raza Naqvi, MBBS, FCPS ${ }^{1}$, Han Naung Tun, MBBS, MD ${ }^{2,3^{*}}$, Abdul Razzaq, MBBS, FCPS , \\ Zubair Zaffar, MBBS, FCPS 4 , Syed Nauman Ali, MBBS, FCPS ${ }^{5}$, Haroon Babar, MBBS, FCPS ${ }^{6}$ and Tariq \\ Abbas, MBBS, FCPS, FRCP
}

\author{
${ }^{1}$ National Institute of Cardiovascular Diseases, Pakistan \\ ${ }^{2}$ Working Group on Aorta and Peripheral Vascular Diseases, European Society of Cardiology, France
}

${ }^{3}$ National Representative of Heart Failure Specialist of Tomorrow for Myanmar in European Heart Failure Association, European Society of Cardiology, France

${ }^{4} \mathrm{CPE}$ Institute of Cardiology, Pakistan

${ }^{5}$ Bahawal Victoria Hospital, Pakistan

${ }^{6}$ Nishtar Medical University and Hospital, Pakistan

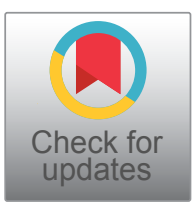

*Corresponding author: Han Naung Tun, MBBS, MD, Working Group on Aorta and Peripheral Vascular Diseases, National Representative of Heart Failure Specialist of Tomorrow for Myanmar in European Heart Failure Association, European Society of Cardiology, Sophia Antipolis, France

\begin{abstract}
Introduction: An abnormal ankle-brachial index (ABI) is associated with higher risk for future cardio-vascular (CV) events; however, it is unknown whether this association is true in patients with associated diabetes mellitus (DM).

Objectives: The objective of this study was to determine frequency of coronary artery disease in terms of number of vessels involved using angiography in diabetic patients with abnormal ankle brachial index.

Subjects and methods: This Cross Sectional Study was done at Department of Adult Cardiology, CPE institute of Cardiology Multan. ABI was measured using the standard method. After measuring the $A B I$, angiography was done on patients with abnormal $\mathrm{ABI}$ and pattern of Coronary Artery Disease $(C A D)$ was recoded noting the number of vessels involved.

Results: A total of 115 patients were included in the study. The mean age of patients was 62.40 years with standard deviation of 2.78 years. Majority of the patients in the study were male $(68.7 \%)$. Mean ABI was of study participants was 1.2 with standard deviation of 0.58 while mean duration of disease was 3.2 years with standard deviation of 0.7 years. 97 of the study participants were hypertensive and 18 were smokers. A total of 28 patients $(24.3 \%)$ did not have CAD
\end{abstract}

as per angiography while the rest had 1 vessel disease $(33.4 \%), 2$ vessel disease $(29.5 \%)$, and 3 vessel disease $(20.9 \%)$. Stratification of patients by age, gender, duration of disease, hypertension and low/high ABI showed $p$ value > 0.05 in all cases showing statistically insignificant difference between various subgroups.

Conclusion: It is concluded that there is high frequency of CAD in diabetic patients with abnormal ABI.

\section{Keywords}

Coronary artery disease, Ankle brachial index, Peripheral arterial disease

\section{Introduction}

Ankle-brachial index (ABI) has been suggested as a useful predictor of generalized atherosclerosis; individuals with a low $A B I$ have an increased risk of death from cardiovascular disease (CVD) in epidemiological studies 1. $A B I$ is an inexpensive and reliable method for detection of peripheral artery disease (PAD), and it has also been proposed as a marker of subclinical atherosclerosis and cardiovascular prognosis [1,2]. PAD caused by

Citation: Naqvi SHR, Tun HN, Razzaq A, Zaffar Z, Ali SN, et al. (2019) Angiographic Pattern of Coronary Artery Disease in Diabetic Patients Having Abnormal Ankle Brachial Index. Int J Clin Cardiol 6:154. doi. org/10.23937/2378-2951/1410154

Accepted: August 07, 2019; Published: August 09, 2019

Copyright: (C) 2019 Naqvi SHR, et al. This is an open-access article distributed under the terms of the Creative Commons Attribution License, which permits unrestricted use, distribution, and reproduction in any medium, provided the original author and source are credited. 
atherosclerotic occlusion in the arteries of the legs, is an important manifestation of systemic atherosclerosis and is defined as an ankle-brachial index $A B I(\leq 0.90)$. Diabetes is a powerful risk factor for PAD, and the measurement of $A B I$ is used to detect PAD in diabetic patients 3. However, in diabetic patients, it is not infrequent to find very stiff and even incompressible ankle arteries, leading to a high $\mathrm{ABI}(>1.40)$. This situation is due to the presence of medial calcinosis (MC), a specific disease distinct from atherosclerosis, corresponding to the calcification of the medial layer, without protrusion into the arterial lumen [3]. Thus, it is important to study the pattern of CAD in diabetic patients with abnormal $A B I$ as the results can be confounded by MC.

This study is prompted to examine the relationship of $A B I$ with vessel involvement and degree of stenosis on angiography in Pakistani diabetic patients with abnormal $A B I$. The present study will help in clarifying the exact burden of the CAD in diabetic patients with abnormal $A B I$. This study, by determining the burden of CAD in diabetic patients with abnormal $A B I$, will be helpful selecting and referring patients for timely angiography thus decreasing mortality in this group of patients with high burden of silent CAD. It is also important to study this because it causes significant disease and financial burden. The study will be a valuable contribution as diabetes is a major public health problem in Pakistan.

\section{Objectives of the study}

The objective of this study was to determine frequency of coronary artery disease in terms of number of vessels involved using angiography in diabetic patients with abnormal ankle brachial index.

\section{Operational Definition}

\section{Ankle brachial index}

$A B I$ was obtained using a standard protocol by placing patients in a supine position for at least 5 minutes. Systolic blood pressure of the brachial artery of both arms and the posterior tibial artery of both ankles was measured using a doppler probe. The highest blood pressure in the arms was be used to calculate ABI. The ratio of ankle to arm systolic blood pressure was calculated for each leg and the lowest ratio was recorded as the $A B I$ for the patient. The $A B I$ results were categorized among one of the following groups: low $A B I(\leq 0.90)$, normal $A B I$ (0.91-1.39), and high $A B I(\geq 1.40)$. $A B I \leq 0.90$ and $\geq 1.40$ was taken as abnormal.

\section{Diabetes mellitus}

Patients were classified as having DM if they are receiving treatment with oral hypoglycemic agents or insulin for more than a year.

\section{Angiographic pattern}

It was assessed on angiography in terms of number of vessels involved viz., 1,2,3.

\section{Materials and Methods}

\section{Study design}

Cross Sectional Study.

\section{Setting}

The study was conducted in Department of Adult Cardiology, CPE institute of Cardiology Multan.

\section{Duration of study}

6 months after approval of synopsis.

\section{From}

01.01.2016 to 30.06 .2016 .

\section{Sample size}

Sample size was calculated to be 115 with $95 \%$ confidence interval, $10 \%$ margin of error and $11.8 \%$ expected frequency of CAD2 (one vessel disease) in diabetic patients abnormal $\mathrm{ABI}$ using WHO sample size calculator.

\section{Sampling technique}

Non probability consecutive sampling.

\section{Sample selection}

Inclusion criteria:

- Diabetic patients with disease duration more than one year as per operational definition

- Both Genders

- Aged 50-70 years

- $\mathrm{ABls}$ of patient in abnormal range low $\leq 0.9$; high $>$ 1.4 .

\section{Exclusion criteria:}

- Patients with unstable cardiopulmonary, neurological, or psychiatric disease based on history and/or previous medical record

- Patients having vascular surgery of lower limbs as determined from medical record and history/ examination.

- Patients not willing to participate in the study.

\section{Data collection procedures}

115 patients with diabetes mellitus who fulfill the inclusion and exclusion criteria were included in the study after taking Informed consent. ABI was measured using the standard method described previously. $A B I \leq 0.90$ and $\geq 1.40$ was taken as abnormal. After measuring the $A B I$, angiography was done on patients with abnormal $A B I$ and pattern of CAD was recoded noting the number of vessels involved and degree of stenosis. Biodata was be entered in a predesigned structured proforma.

\section{Statistical analysis}

Statistical analysis was done using Statistical Package 
for Social Sciences (SPSS) version 16. Qualitative data like gender, presence of $C A D$, low/high $A B I$, hypertension, smoking history were presented as frequencies and percentages. Quantitative data i.e., age, $A B I$, duration of disease, number of vessel involved, were presented as means and standard deviations. Effect modifiers like age, gender, duration of disease, hypertension, low/high $A B I$, were dealt with through stratification. Post stratification chi square test was applied with $p$ value $<0.05$ was taken statistically significant.

\section{Results}

A total of 115 patients were included in the study. The mean age of patients was 62.40 years with standard

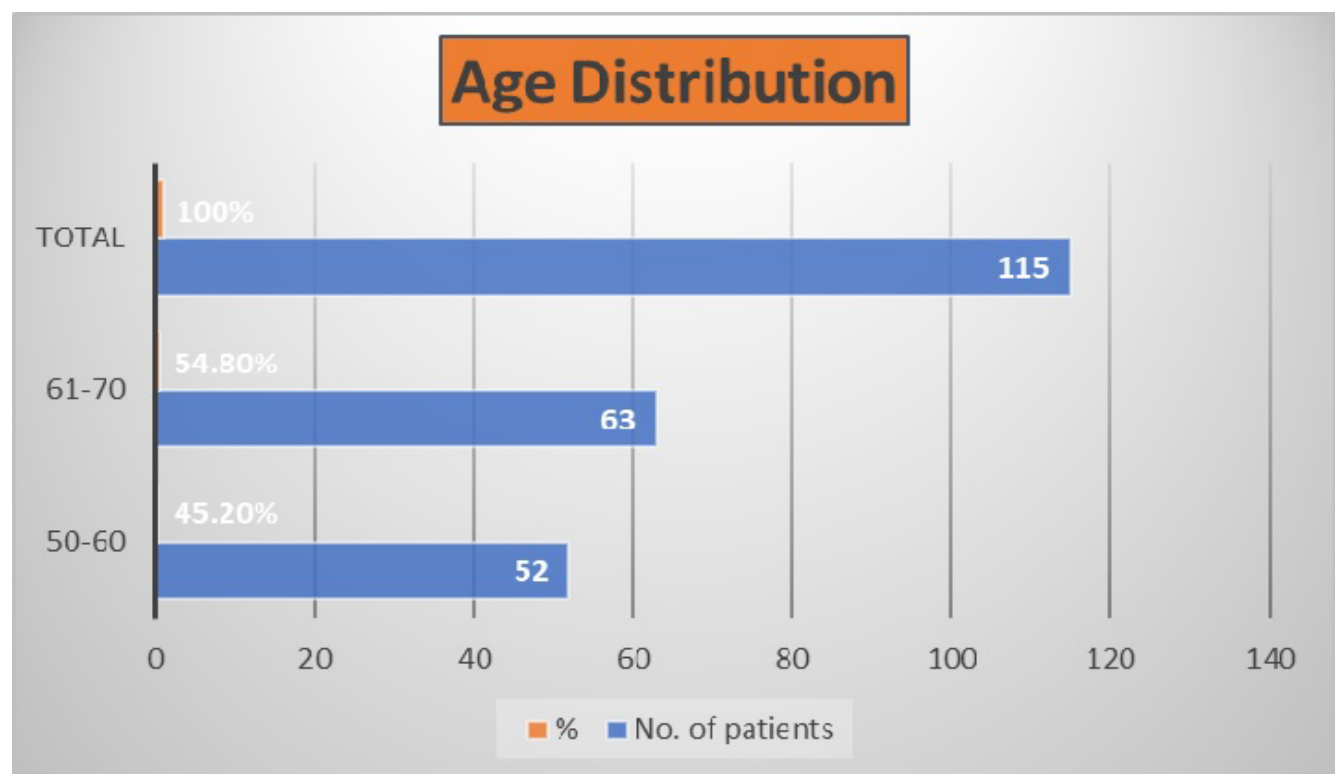

Figure 1: Age distribution, $n=115$, Mean \pm SD: $62.40 \pm 2.78$ years.

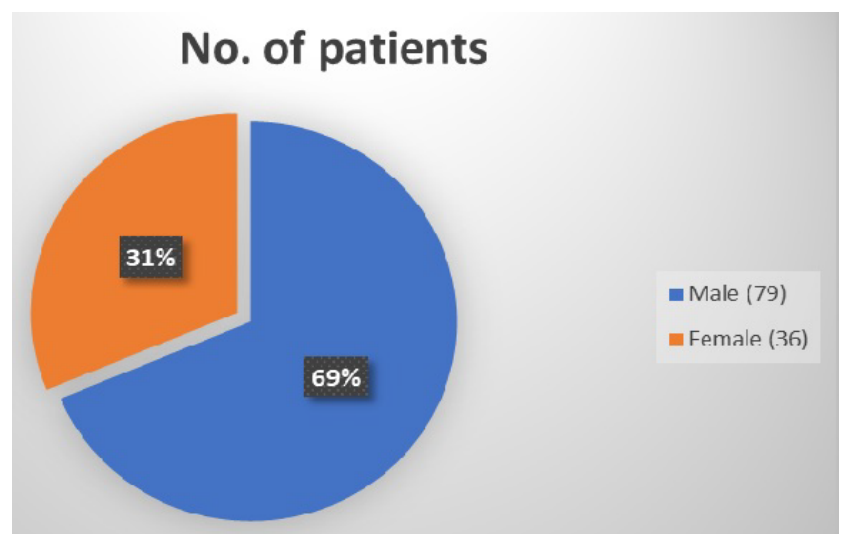

Figure 2: Gender distribution, $\mathrm{n}=115$.

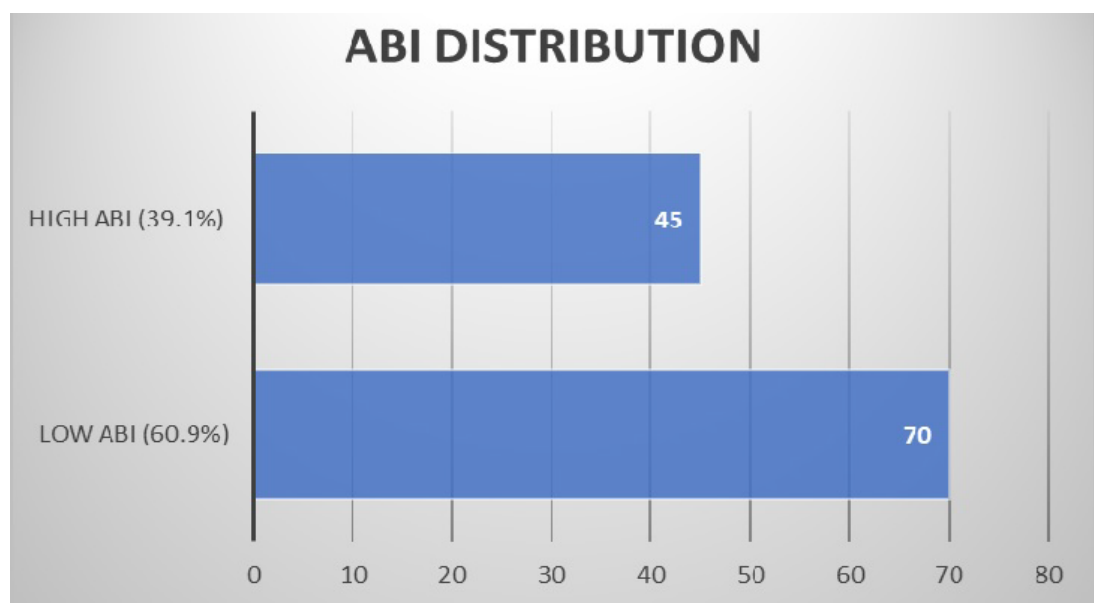

Figure 3: $A B I$ distribution, $n=115$, Mean \pm SD: $1.2 \pm 0.58$. 


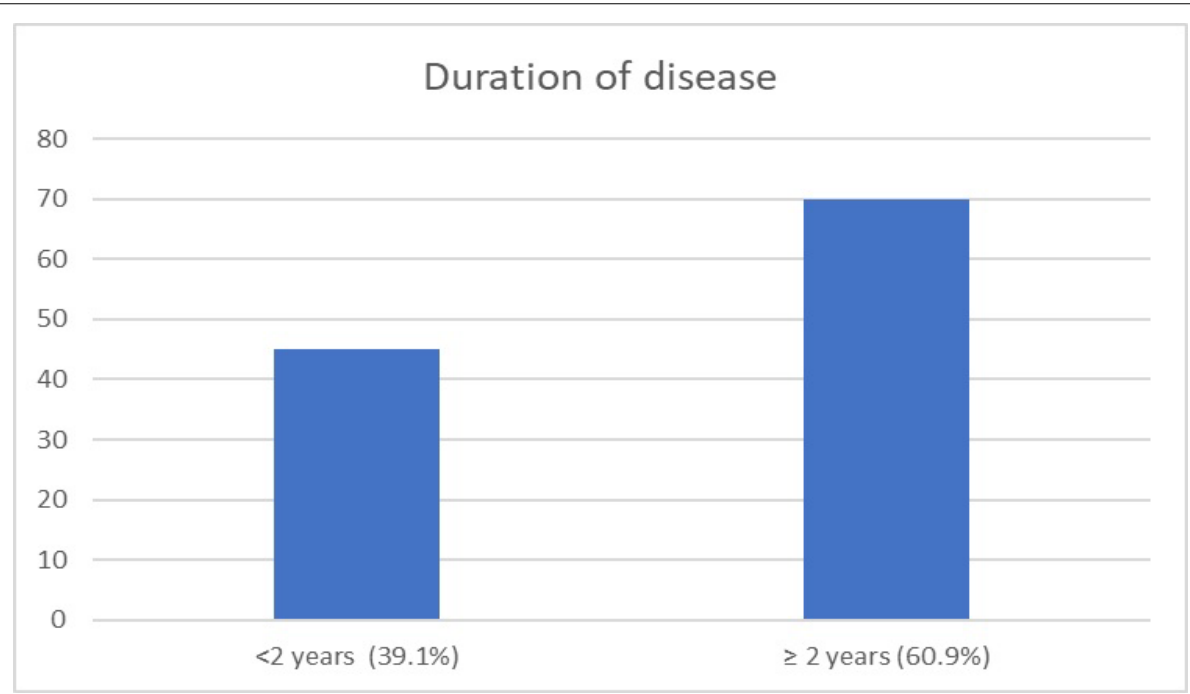

Figure 4: Duration of disease, $n=115$, Mean $\pm S D$ : $3.2 \pm 0.7$ years.

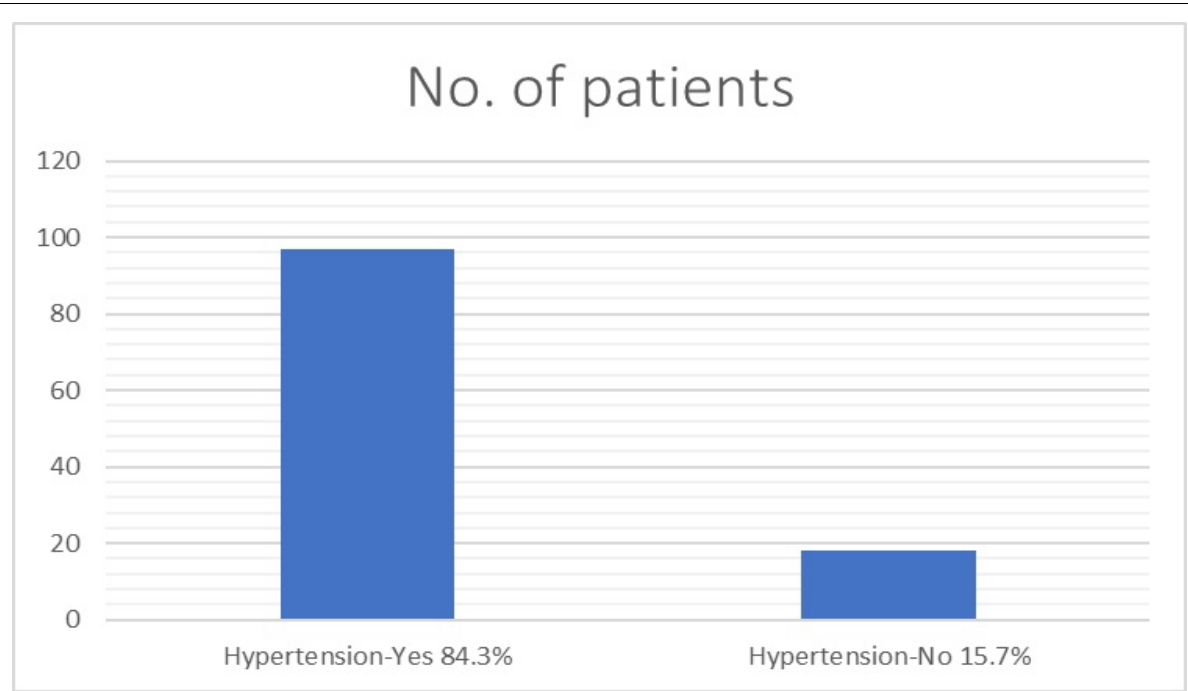

Figure 5: Frequency of hypertension, $n=115$.

\section{Hypertension, Smoking and CAD}

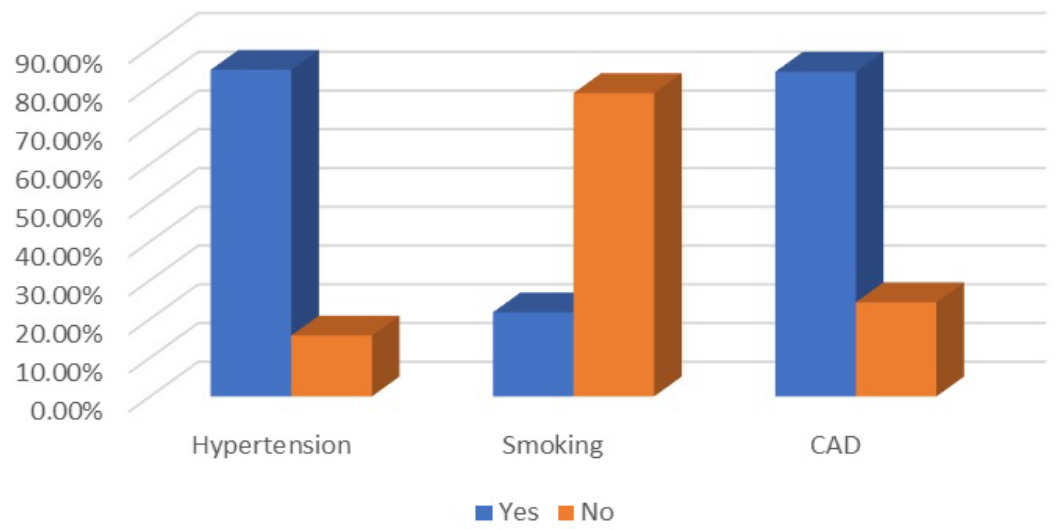

Figure 6: Combination of Figure 5, Figure 6, Figure $7(n=115)$.

deviation of 2.78 years with most of the patients in the age range $61-70$ years. Age distribution of the patients is given in Figure 1. Majority of the patients in the study were male (68.7\%). Gender distribution is outlined in
Figure 2. Mean $A B I$ of study participants was 1.2 with standard deviation of 0.58 . Classification of $A B I$ is given in Figure 3. Mean duration of disease was 3.2 years with standard deviation of 0.7 years, outlines in Figure 
Table 1: Combination of Figure 1, Figure 2, Figure 3, Figure 4.

\begin{tabular}{|l|l|l|l|l|}
\hline Category & Interval & No. & $\%$ & Mean + SD \\
\hline \multirow{2}{*}{ Age in years } & $50-60$ & 52 & $45.20 \%$ & $62.40 \pm 2.78$ years \\
\hline \multirow{2}{*}{ Gender } & $61-70$ & 63 & $54.80 \%$ & \\
\hline \multirow{2}{*}{ ABI } & Male & 79 & $68.70 \%$ & \\
\hline & Female & 36 & $31.30 \%$ & \\
\hline \multirow{2}{*}{ Duration of disease } & Low ABI & 70 & $60.90 \%$ & Mean \pm SD: $1.2 \pm 0.58$ \\
\hline & High ABI & 45 & $39.10 \%$ & Mean \pm SD: $3.2 \pm 0.7$ years \\
\hline
\end{tabular}

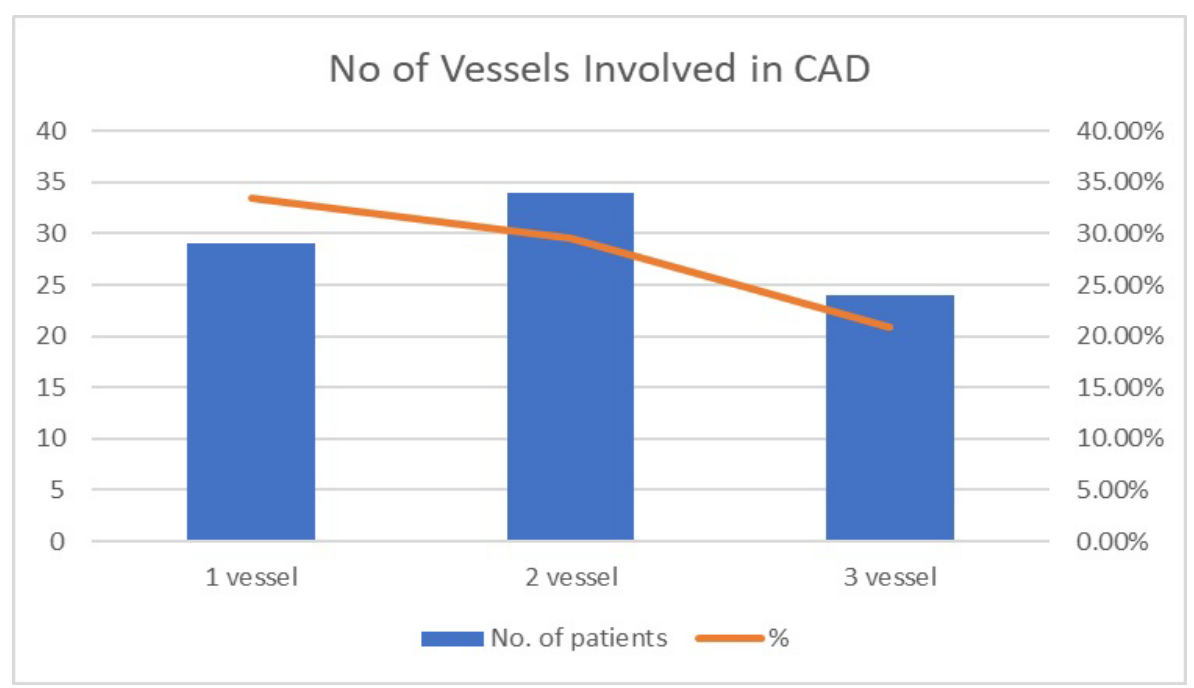

Figure 7: No. of vessel involved in CAD, mean number of vessels involved $1.76 \pm 0.32$

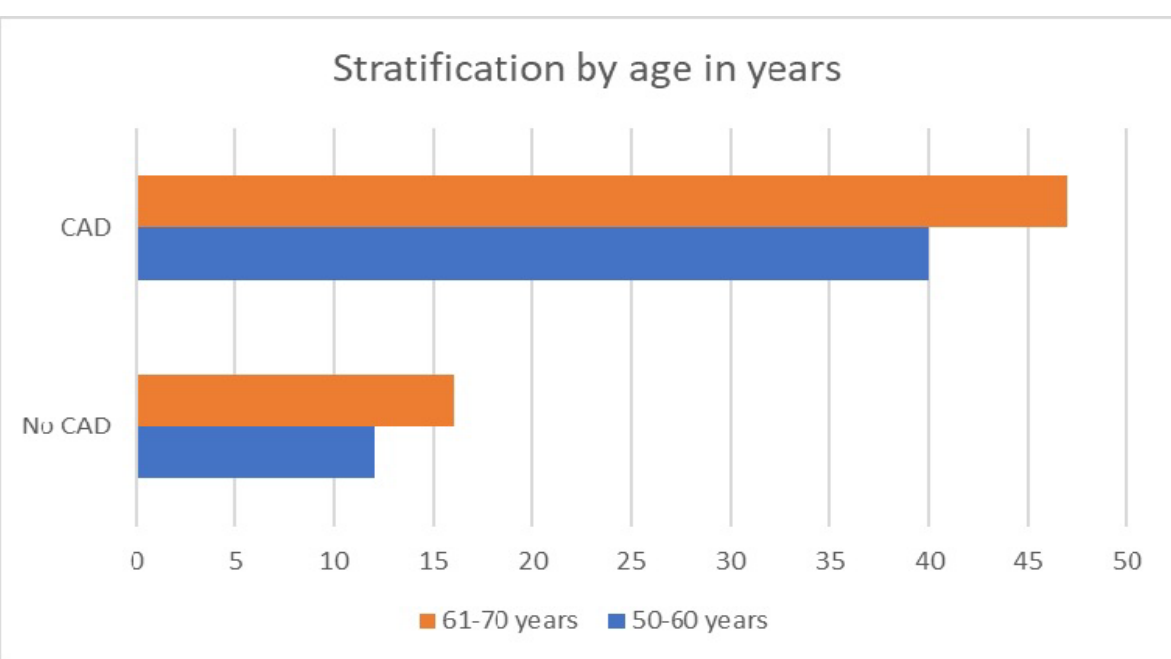

Figure 8: Stratification by age $(n=115)$.

Chi Square Test, Value: 0.258 , Df: 3 , P value: 0.878 .

4, Table 1 . The 97 of the study participants were hypertensive and 18 were smokers, given in Figure 5, Figure 6 respectively.

A total of 28 patients (24.3\%) did not have CAD as per angiography while the rest had 1 vessel disease (33.4\%), 2 vessel disease (29.5\%), and 3 vessel disease $(20.9 \%)$ given in Figure 7 . Stratification of patients by age, gender, and duration of disease, hypertension and low/high $A B I$ is given in Figure 8, Figure 9, Figure 10,
Figure 11, Figure 12 and Figure 13 respectively. $P$ value was $>0.05$ in all cases showing statistically insignificant difference between various subgroups.

\section{Discussion}

The findings of this study are in agreement with some previous studies and reinforce the importance of screening for $A B I$ and $C A D$ in diabetic patients.

The findings of our study underscore the facts that 


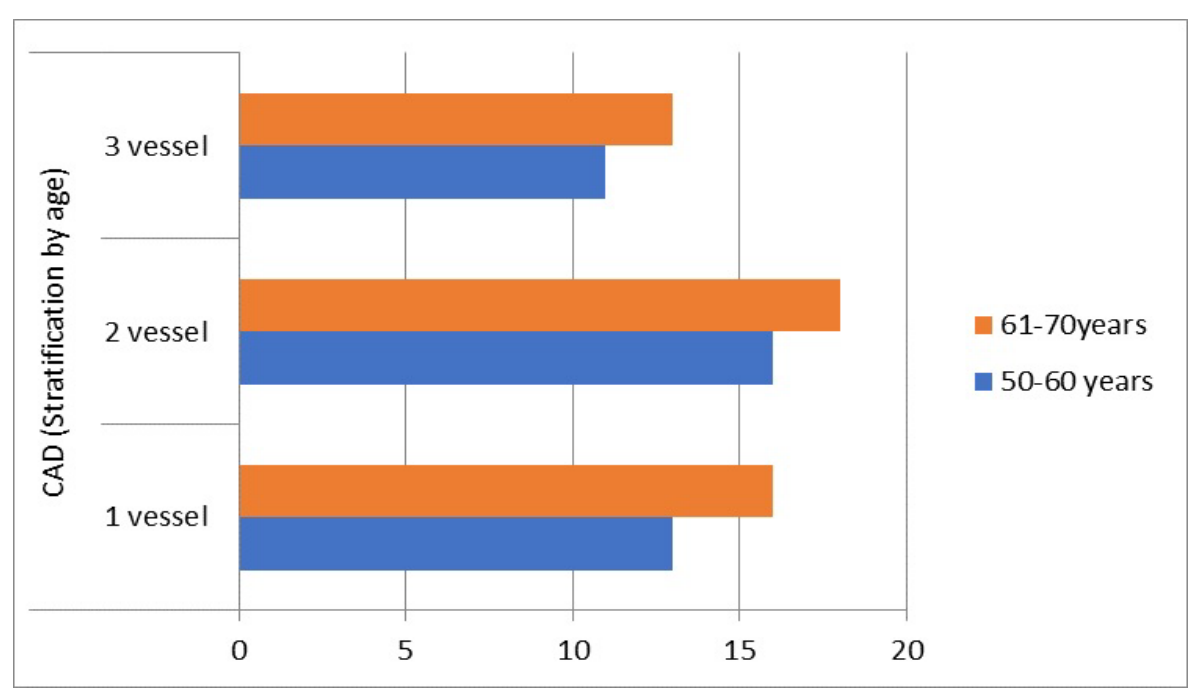

Figure 9: No. of vessels involved, stratification by age.

Chi Square Test, Value: 0.085 , Df: 3, P value: 0.770 .

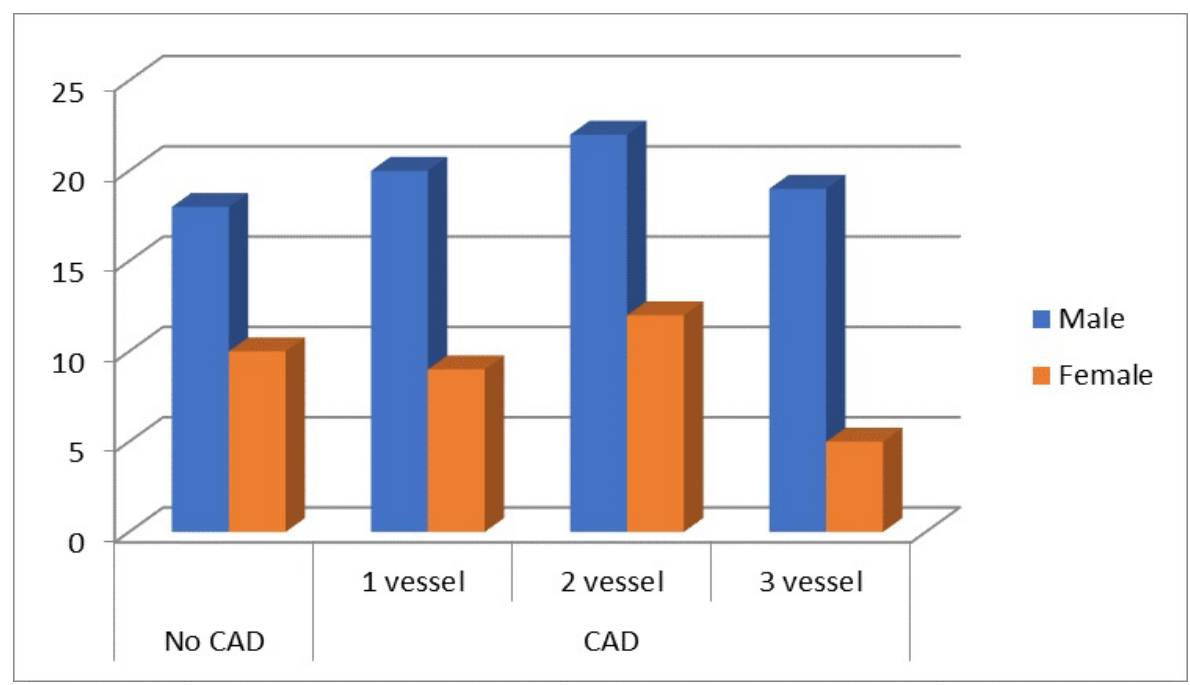

Figure 10: Stratification by gender.

Chi Square Test, Value: 0.019, Df: 3, P value: 0.891 .

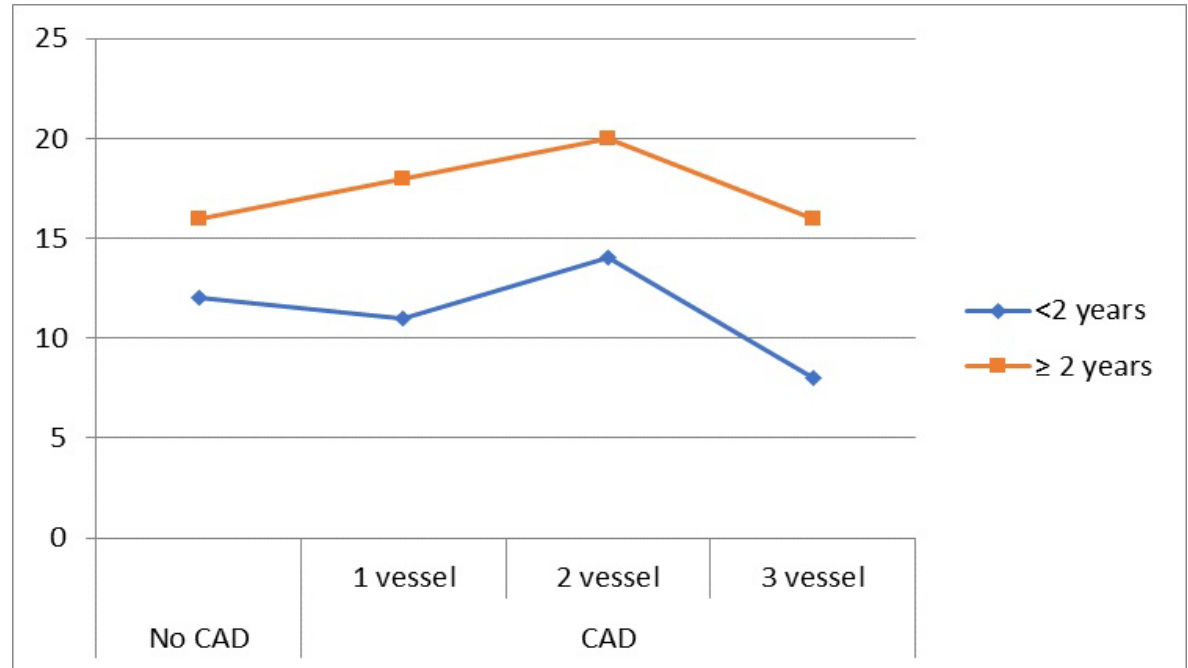

Figure 11: Stratification by duration of diseases.

there is significant overlap between CAD and PAD and that the identification of PAD in patients with stable
CAD may provide important prognostic information in addition to the presence of DM, an independent 


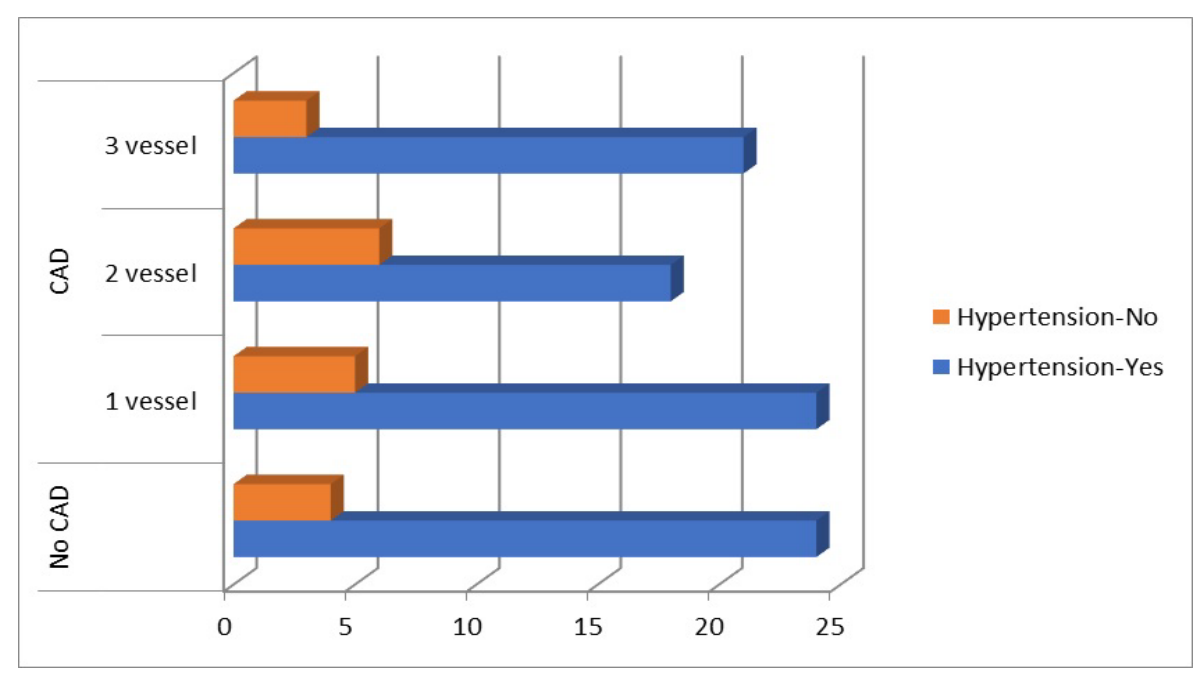

Figure 12: Stratification by hypertension, $n=115, P=0.804$.

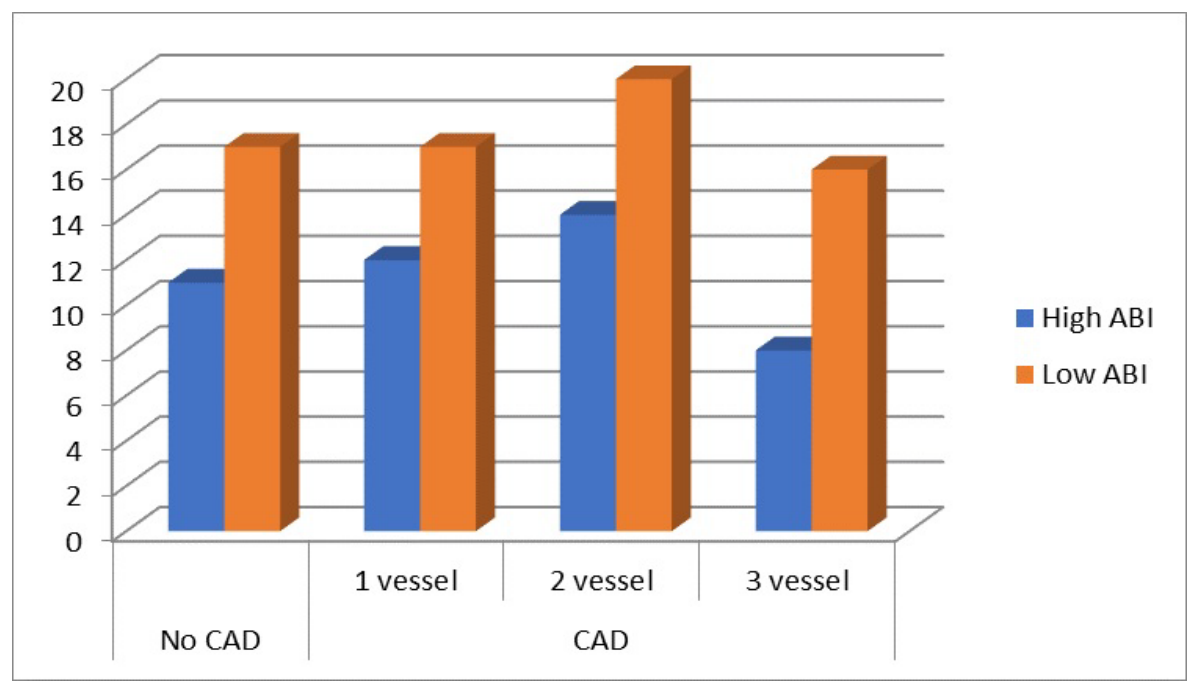

Figure 13: Stratification by low/high $A B I, n=115, P=0.770$.

and important risk factor for death and myocardial infarction.

The overlap between PAD and CAD has been established on the basis of various population-based studies [3-5]. Although the prevalence and outcomes of associated CAD in patients with PAD are well defined, there are limited data on the prevalence and prognostic significance of establishing the diagnosis of PAD in patients with stable CAD. Studies that have investigated the predictive value of $A B I$ in a patient cohort with CAD are scarce. Dieter, et al. reported a $40 \%$ prevalence of PAD in hospitalized patients with CAD and the feasibility of performing an $A B I$ in that setting [6]. Grenon, et al. reported a $70 \%$ increased risk of subsequent $\mathrm{CV}$ events (adjusted HR 1.7, 95\% $\mathrm{Cl} 1.0$ to $2.9, \mathrm{p} 1 / 40.04$ ) and an $80 \%$ increased risk of death (adjusted HR 1.8, 95\% $\mathrm{Cl} 1.2$ to $2.7, \mathrm{p} 1 / 40.006$ ) after adjusting for traditional risk factors [7]. Evidence from the Strong Heart Study identified the association between an abnormal $\mathrm{ABI}$ and mortality, highlighting a U-shaped relation between this noninvasive measure of PAD and mortality risk [8]. It also identified the cutoff values of abnormal $\mathrm{ABI}(<0.9$ and $>1.4$ ), adopted by the American College of Cardiology (ACC) and American Heart Association (AHA) PAD guidelines and also used in our analysis [9]. Evidence from the Coronary Artery Surgery Study indicated that in patients with CAD, presence of PAD had a $25 \%$ greater likelihood of mortality than patients without (multivariate chi-square $25.83, \mathrm{HR} 1.25,95 \% \mathrm{Cl} 1.15$ to 1.36 , $\mathrm{p}<0.001$ ) [10].

The increased vulnerability to $C V$ events in patients with CAD with an abnormal $A B I$ can be partially explained by shared CV risk factors and excess inflammation indicated by significantly higher serum levels of inflammatory bio-markers in this patient population $[11,12]$. The higher repeat coronary events and revascularization rate in patients with CAD and PAD, observed in our study, can be potentially attributed to the significantly greater constrictive remodeling and plaque progression of atherosclerotic coronary arteries in patients with PAD.

In a recent study Mostaza, et al. reported that in patients with $C V$ disease, $A B I$ is a good predictor of the risk 
of recurrent $\mathrm{CV}$ events and death, however, only in subjects without DM [13]. The investigators argued that the presence of DM in patients with already established CV disease in itself confers such a high risk that an abnormal $A B I$ does not add any prognostic value. As the CV outcomes in patients with CAD are strongly influenced by secondary prevention strategies, the findings from our study conducted in the current era of CV care may be of greater relevance to current practice. Many of the earlier studies were either conducted in highly selective patient populations, such as the Strong Heart Study in American Indians, or did not include an $A B I$ in defining the presence of PAD. Exclusive reliance for establishing the diagnosis of PAD on the basis of prior cerebrovascular and/or other noncoronary peripheral arterial events or interventions could not only underestimate the prevalence of PAD but also limit the significance of such studies. The findings of our study and that of others on the predictive value of $A B I$ in patients with $C A D$ should be clearly distinguished from those conducted in at-risk patient populations, where there is inadequate evidence that an $A B I$ test result provided additional information to predict CV events, beyond the Framingham risk score [14]. Further, the US Preventive Services Task Force recommendation suggested that research is needed to identify potential high-risk subgroups who might benefit from $A B I$ screening [15].

\section{Conclusion}

It is concluded that there is high frequency of $C A D$ in diabetic patients with abnormal ABI. Further studies are needed to determine the risk conferred by this high prevalence of CAD in this age group and steps taken to decrease the associated morbidity and mortality.

\section{References}

1. Singh PP, Abbott JD, Lombardero MS, Sutton-Tyrrell K, Woodhead G, et al. (2011) The prevalence and predictors of an abnormal ankle-brachial index in the bypass angioplasty revascularization investigation 2 diabetes (BARI 2D) trial. Diabetes Care 34: 464-467.

2. Quiles J, Morillas P, Bertomeu V, Mazon P, Cordero A, et al. (2011) Combination of ankle brachial index and diabetes mellitus to predict cardiovascular events and mortality after an acute coronary syndrome. Int J Cardiol 151: 84-88.

3. Barrett-Connor E, Orchard TJ (1985) Insulin-dependent diabetes mellitus and ischemic heart disease. Diabetes Care 1: 65-70.
4. Booth GL, Kapral MK, Fung K, Tu JV (2006) Relation between age and cardiovascular disease in men and women with diabetes compared with non-diabetic people: A population-based retrospective cohort study. Lancet 368: 29-36.

5. Robertson WB, Strong JP (1968) Atherosclerosis in persons with hypertension and diabetes mellitus. Lab Invest 18: 538-551.

6. Waller BF, Palumbo PJ, Lie JT, Roberts WC (1980) Status of the coronary arteries at necropsy in diabetes mellitus with onset after age 30 years. Analysis of 229 diabetic patients with and without clinical evidence of coronary heart disease and comparison to 183 control subjects. Am J Med 69: 498-506

7. Pajunen P, Taskinen MR, Nieminen MS, Syvänne M (2000) Angiographic severity and extent of coronary artery disease in patients with type 1 diabetes mellitus. Am J Cardiol 86: 1080-1085.

8. Stein B, Weintraub WS, Gebhart SP, Cohen-Bernstein CL, Grosswald R, et al. (1995) Influence of diabetes mellitus on early and late outcome after percutaneous transluminal coronary angioplasty. Circulation 91: 979-989.

9. Granger CB, Califf RM, Young S, Candela R, Samaha J, et al. (1993) Outcome of patients with diabetes mellitus and acute myocardial infarction treated with thrombolytic agents. The Thrombolysis and Angioplasty in Myocardial Infarction (TAMI) Study Group. J Am Coll Cardiol 21: 920925.

10. Mueller HS, Cohen LS, Braunwald E, S Forman , F Feit, et al. (1992) Predictors of early morbidity and mortality after thrombolytic therapy of acute myocardial infarction. Analyses of patient subgroups in the Thrombolysis in Myocardial Infarction (TIMI) trial, phase II. Circulation 85: 1254-1264.

11. Natali A, Vichi S, Landi P, Severi S, L'Abbate A, et al. (2000) Coronary atherosclerosis in Type II diabetes: angiographic findings and clinical outcome. Diabetologia 43: 632-641.

12. Melidonis A, Dimopoulos V, Lempidakis E, Hatzissavas J, Kouvaras G, et al. (1999) Angiographic study of coronary artery disease in diabetic patients in comparison with nondiabetic patients. Angiology 50: 997-1006.

13. C Lahoz, JM Mostaza (2006) Ankle-brachial index: A useful tool for stratifying cardiovascular risk. Rev Esp Cardiol 59: 647-649.

14. Pajunen P, Nieminen MS, Taskinen MR, Syvänne M (1997) Quantitative comparison of angiographic characteristics of coronary artery disease in patients with noninsulindependent diabetes mellitus compared with matched nondiabetic control subjects. Am J Cardiol 80: 550-556.

15. Scognamiglio $R$, Negut $C$, Ramondo A, Tiengo A, Avogaro A (2006) Detection of coronary artery disease in asymptomatic patients with type 2 diabetes mellitus. J Am Coll Cardiol 47: 65-71. 